Korean J. Math. 20 (2012), No. 2, pp. 177-184

\title{
A NOTE ON FOUR TYPES OF REGULAR RELATIONS
}

\author{
H. S. SONG
}

\begin{abstract}
In this paper, we study the four different types of relations, $\mathcal{P}(X, T), \mathcal{R}(X, T), \mathcal{L}(X, T)$, and $\mathcal{S}(X, T)$ in a transformation $(X, T)$, and obtain some of their properties. In particular, we give a relationship between $\mathcal{R}(X, T)$ and $\mathcal{S}(X, T)$.
\end{abstract}

\section{Introduction}

The proximal relation were first studied by Ellis and Gottschalk in [6]. The syndetically proximal relation were introduced by Clay in [3]. In [1], Auslander defined the regular minimal sets which may be described as minimal subsets of enveloping semigroups. In [8], Shoenfeld introduced the regular homomorphisms which are defined by extending regular minimal sets to homomorphisms with minimal range. Also $\mathrm{Yu}$ introduced the regular relation and the syndetically regular relation (see $[9],[10])$.

In this paper, we study the four different types of relations in a transformation and give some of their properties.

\section{Preliminaries}

A transformation group $(X, T)$ will consist of a jointly continuous action of the topological group $T$ on the compact Hausdorff space $X$. The group $T$, with identity $e$, is assumed to be topologically discrete and remain fixed throughout this paper, so we may write $X$ instead of $(X, T)$.

Received March 17, 2012. Revised June 2, 2012. Accepted June 5, 2012.

2010 Mathematics Subject Classification: 37B05.

Key words and phrases: proximal relation, regular relation, syndetically proximal relation, syndetically regular relation.

The present research has been conducted by the Research Grant of Kwangwoon University in 2011. 
A flow is said to be minimal if every point has dense orbit. Minimal flows are also referred to as minimal sets.

A homomorphism of transformation groups is a continuous, equivariant map. A one-one homomorphism of $X$ onto $X$ is called an automorphism of $X$. We denote the group of automorphisms of $X$ by $A(X)$.

The compact Hausdorff space $X$ carries a natural uniformity whose indices are the neighborhoods of the diagonal in $X \times X$. Two points $x, x^{\prime} \in X$ are said to be proximal if, given any index $\alpha$, there exists $t \in T$ such that $\left(x t, x^{\prime} t\right) \in \alpha$. The proximal relation in $X$, denoted by $\mathcal{P}(X, T)$, is the set of all proximal pairs in $X . X$ is said to be distal if $\mathcal{P}(X, T)=\triangle_{X}$, the diagonal of $X \times X$ and is said to be proximal if $\mathcal{P}(X, T)=X \times X$.

Given a transformation group $(X, T)$, we may regard $T$ as a set of self-homeomorphisms of $X$. We define $E(X)$, the enveloping semigroup of $X$ to be the closure of $T$ in $X^{X}$, taken with the product topology. $E(X)$ is at once a transformation group and a sub-semigroup of $X^{X}$. The minimal right ideals of $E(X)$, considered as a semigroup, coincide with the minimal sets of $E(X)$. A subset $A$ of $T$ is said to be syndetic if there exists a compact subset $K$ of $T$ with $T=A K$.

Two points $x, x^{\prime} \in X$ are said to be syndetically proximal if, given any index $\alpha$, there exists a syndetic subset $A$ of $T$ such that $\left(x t, x^{\prime} t\right) \in \alpha$ for all $t \in A$. The set of syndetically proximal pairs in $X$ is called the syndetically proximal relation and is denoted by $\mathcal{L}(X, T)$.

Two points $x, x^{\prime} \in X$ are said to be regular if there exists $h \in A(X)$ such that $\left(h(x), x^{\prime}\right) \in \mathcal{P}(X, T)$. The set of regular pairs in $X$ is called the regular relation and is denoted by $\mathcal{R}(X, T)$.

Two points $x, x^{\prime} \in X$ are said to be syndetically regular if there exists $h \in A(X)$ such that $\left(h(x), x^{\prime}\right) \in \mathcal{L}(X, T)$. The set of syndetically regular pairs in $X$ is called the syndetically regular relation and is denoted by $\mathcal{S}(X, T)$.

$X$ is said to be almost periodic if, given any index $\alpha$, there exists a syndetic subset $A$ of $T$ such that $x A \subset x \alpha$ for all $x \in X$, where $x \alpha=\{y \in X \mid(x, y) \in \alpha\} . X$ is said to be locally almost periodic if, given $x \in X$ and $U$ a neighborhood of $x$, there exists a neighborhood $V$ of $x$ and a syndetic subset $A$ of $T$ with $V A \subset U$.

REMARK 2.1. If $E(X)$ contains just one minimal right ideal, then $\mathcal{P}(X, T)$ and $\mathcal{R}(X, T)$ are invariant equivalence relations on $X$ (see [4], $[9])$. 
Lemma 2.2. ([2]) Suppose $(X, T)$ is locally almost periodic. Then $\mathcal{P}(X, T)=\mathcal{L}(X, T)$.

Lemma 2.3. ([4]) $(X, T)$ is almost periodic iff it is locally almost periodic and distal.

\section{Some results on $\mathcal{P}(X, T), \mathcal{R}(X, T), \mathcal{L}(X, T)$ and $\mathcal{S}(X, T)$}

The following lemma is an immediate consequence of the definitions.

Lemma 3.1. Given a transformation group $(X, T)$, the following statements are true :

(1) $\mathcal{L}(X, T) \subset \mathcal{P}(X, T) \subset \mathcal{R}(X, T)$.

(2) $\mathcal{L}(X, T) \subset \mathcal{S}(X, T) \subset \mathcal{R}(X, T)$.

(3) $\triangle_{X} \subset \mathcal{L}(X, T)$

(4) If $\mathcal{P}(X, T)=\mathcal{L}(X, T)$, then $\mathcal{R}(X, T)=\mathcal{S}(X, T)$.

The next lemma leads to a useful characterization of $\mathcal{L}(X, T)$.

Lemma 3.2. ([5]) Given a transformation group $(X, T)$, the following statements are true :

(1) $\mathcal{L}(X, T)=\{(x, y) \in X \times X \mid \overline{(x, y) T} \subset \mathcal{P}(X, T)\}$.

(2) $\mathcal{L}(X, T)$ is an invariant equivalence relation on $X$.

Lemma 3.3. Given a transformation group $(X, T)$, the following statements are true:

(1) $\mathcal{S}(X, T)=\{(x, y) \in X \times X \mid \overline{(x, y) T} \subset \mathcal{R}(X, T)\}$.

(2) If $E(X)$ contains just one minimal right ideal, then $\mathcal{S}(X, T)$ is an invariant equivalence relation on $X$.

Proof. (1) Use lemma 3.2(1). Assume that $(x, y) \in X \times X$. Then $(x, y) \in \mathcal{S}(X, T)$ iff there exists $h \in A(X)$ such that $(h(x), y) \in \mathcal{L}(X, T)$ iff there exists $h \in A(X)$ such that $\overline{(h(x), y) T} \subset \mathcal{P}(X, T)$ iff there exists $h \in A(X)$ such that $(h(x p), y p) \in \mathcal{P}(X, T)$ for all $p \in E(X)$ iff $(x, y) p \in$ $\mathcal{R}(X, T)$ for all $p \in E(X)$ iff $\overline{(x, y) T} \subset \mathcal{R}(X, T)$. This completes the proof of $(1)$.

(2) It follows immediately from (1) that $\mathcal{S}(X, T)$ is a reflexive, symmetric and invariant relation. To see that $\mathcal{S}(X, T)$ is transitive, assume that $(x, y) \in \mathcal{S}(X, T)$ and $(y, z) \in \mathcal{S}(X, T)$. Then $\overline{(x, y) T} \subset \mathcal{R}(X, T)$ and $\overline{(y, z) T} \subset \mathcal{R}(X, T)$ and hence $(x p, y p) \in \mathcal{R}(X, T)$ and $(y p, z p) \in$ 
$\mathcal{R}(X, T)$ for all $p \in E(X)$. Since $E(X)$ contains just one minimal right ideal, we have from Remark 2.1 that $(x p, z p) \in \mathcal{R}(X, T)$ for all $p \in E(X)$. Therefore $\overline{(x, z) T} \subset \mathcal{R}(X, T)$ and hence $(x, z) \in \mathcal{S}(X, T)$.

REMARK 3.4. $\mathcal{P}(X, T), \mathcal{R}(X, T)$, and $\mathcal{S}(X, T)$ are not equivalence relations on $X$. However, if $E(X)$ contains just one minimal right ideal, then they are invariant equivalence relations on $X$ (see Remark 2.1 and Lemma 3.3).

Lemma 3.5. If $\mathcal{P}(X, T)$ is closed, then $\mathcal{R}(X, T)$ is also closed.

Proof. Let $(x, y) \in \mathcal{R}(X, T)$ and let $q \in E(X)$. Then there exists $h \in A(X)$ such that $(h(x), y) \in \mathcal{P}(X, T)$. Since $\mathcal{P}(X, T)$ is closed, we have that $(h(x), y) q \in \mathcal{P}(X, T)$ and therefore $(h(x q), y q) \in \mathcal{P}(X, T)$. This implies that $(x q, y q) \in \mathcal{R}(X, T)$. Thus $\mathcal{R}(X, T)$ is closed.

Theorem 3.6. Let $\mathcal{P}(X, T)$ be closed. Then

(1) $\mathcal{P}(X, T)=\mathcal{L}(X, T)$

(2) $\mathcal{R}(X, T)=\mathcal{S}(X, T)$

Proof. To see that (1) holds, assume that $(x, y) \in \mathcal{P}(X, T)$. Since $\mathcal{P}(X, T)$ is closed, it follows that $\overline{(x, y) T} \subset \mathcal{P}(X, T)$. By Lemma 3.2(1), it follows that $\mathcal{P}(X, T) \subset \mathcal{L}(X, T)$ and therefore $\mathcal{P}(X, T)=\mathcal{L}(X, T)$.

The proof of $(2)$ is exactly analogous to that of (1) by Lemma 3.5.

Ellis' result [4, Lemma 5.17] is a corollary to the above theorem.

Corollary 3.7. Let $\mathcal{P}(X, T)$ be closed. Then it is an invariant equivalence relation on $X$.

REMARK 3.8. Let $(X, T)$ is distal. Since $\mathcal{P}(X, T)=\triangle_{X}$, it follows that $\mathcal{L}(X, T)=\mathcal{P}(X, T)$ and therefore $\mathcal{P}(X, T)$ is a closed invariant equivalence relation on $X$ (see [4, Lemma 5.12]).

We can prove Ellis' result [4, Lemma 5.27] as follows :

Theorem 3.9. Suppose $(X, T)$ is locally almost periodic. Then the following statements are true :

(1) $\mathcal{L}(X, T)=\mathcal{P}(X, T) \subset \mathcal{R}(X, T)=\mathcal{S}(X, T)$.

(2) $\mathcal{P}(X, T)$ and $\mathcal{R}(X, T)$ are closed invariant equivalence relations on $X$.

Proof. (1) This follows from Lemma 2.2 and Lemma 3.1(4). 
(2)] The fact that $\mathcal{P}(X, T)$ is an invariant equivalence relation on $X$ follows from (1) and Lemma 3.2(2). Since $\mathcal{P}(X, T)$ is transitive, it follows from [4, Proposition 5.16] that $E(X)$ contains just one minimal right ideal and therefore $\mathcal{R}(X, T)$ is an invariant equivalence relation on $X$ by Remark 2.1. The closed property of $\mathcal{P}(X, T)$ follows from [4, Proposition 5.26]. The closed property of $\mathcal{R}(X, T)$ follows from Lemma 3.5.

The proof of the following corollary follows immediately from Lemma 2.3 .

Corollary 3.10. Suppose $(X, T)$ is almost periodic. Then the following statements are true :

(1) $\mathcal{L}(X, T)=\mathcal{P}(X, T) \subset \mathcal{R}(X, T)=\mathcal{S}(X, T)$.

(2) $\mathcal{P}(X, T)$ and $\mathcal{R}(X, T)$ are closed invariant equivalence relations on $X$.

Theorem 3.11. Suppose $A(X)=\left\{1_{X}\right\}$, where $\left\{1_{X}\right\}$ is the identity homomorphism of $X$. Then $\mathcal{L}(X, T)=\mathcal{S}(X, T) \subset \mathcal{P}(X, T)=\mathcal{R}(X, T)$.

Proof. Let $(x, y) \in \mathcal{S}(X, T)$. Then $\overline{(x, y) T} \subset \mathcal{R}(X, T)$ by Lemma 3.3(1). Since $A(X)=\left\{1_{X}\right\}$, it follows that $\mathcal{P}(X, T)=\mathcal{R}(X, T)$ and hence $(x, y) \in \mathcal{L}(X, T)$ by Lemma 3.2(1). Therefore $\mathcal{S}(X, T)=\mathcal{L}(X, T)$.

Corollary 3.12. Suppose $(X, T)$ is minimal and proximal. Then $\mathcal{L}(X, T)=\mathcal{S}(X, T) \subset \mathcal{P}(X, T)=\mathcal{R}(X, T)$.

Proof. The proof uses [7, (8) of Section 1] to show that if $(X, T)$ is minimal and proximal, then the only homomorphism $(X, T) \rightarrow(X, T)$ is the identity.

Lemma 3.13. Let $h \in A(X)$ and let $\check{h}: X \times X \rightarrow X \times X$ be the map induced by $h$. Then the following statements are true:

(1) $\check{h} \mathcal{P}(X, T) \subset \mathcal{P}(X, T)$.

(2) $\check{h} \mathcal{R}(X, T) \subset \mathcal{R}(X, T)$.

(3) $\breve{h} \mathcal{L}(X, T) \subset \mathcal{L}(X, T)$.

(4) $\breve{h} \mathcal{S}(X, T) \subset \mathcal{S}(X, T)$.

Proof. The proof of (1) is analogous to that of [4, Proposition 5.22]. Let $(x, y) \in \mathcal{R}(X, T)$. Then there exists $\psi \in A(X)$ with $(\psi(x), y) \in$ 
$\mathcal{P}(X, T)$. By (1) $(h \circ \psi(x), h(y))=\left(h \circ \psi \circ h^{-1} \circ h(x), h(y)\right) \in \mathcal{P}(X, T)$. Since $h \circ \psi \circ h^{-1} \in A(X)$, it follows that $(h(x), h(y))=\check{h}(x, y) \in \mathcal{R}(X, T)$. Now let $(x, y) \in \mathcal{L}(X, T)$. Then $\overline{(x, y) T} \subset \mathcal{P}(X, T)$ by Lemma 3.2(1), which means that $(x, y) p \in \mathcal{P}(X, T)$ for all $p \in E(X)$. By $(1) \check{h}(x, y) p \in$ $\mathcal{P}(X, T)$ for all $p \in E(X)$. Therefore $\overline{(h(x), h(y)) T} \subset \mathcal{P}(X, T)$ and hence $(h(x), h(y)) \in \mathcal{L}(X, T)$. This proves that $\breve{h} \mathcal{L}(X, T) \subset \mathcal{L}(X, T)$. The proof of (4) is analogous to that of (3).

Theorem 3.14. Let $h \in A(X)$ and let $\check{h}: X \times X \rightarrow X \times X$ be the map induced by $h$. Then the following statements are true :

(1) If $(X, T)$ is minimal, then $\check{h} \mathcal{P}(X, T)=\mathcal{P}(X, T)$.

(2) If $(X, T)$ is minimal and $A(X)$ is abelian, then $\breve{h} \mathcal{R}(X, T)=\mathcal{R}(X, T)$.

Proof. If $(X, T)$ is minimal, then it is pointwise almost periodic. Thus (1) follows from [4, Proposition 5.22]. To see (2), let $\left(y_{1}, y_{2}\right) \in \mathcal{R}(X, T)$. Then there exists $\psi \in A(X)$ with $\left(\psi\left(y_{1}\right), y_{2}\right) \in \mathcal{P}(X, T)$. By (1) there exists $\left(x_{1}, x_{2}\right) \in \mathcal{P}(X, T)$ such that $\breve{h}\left(x_{1}, x_{2}\right)=\left(\psi\left(y_{1}\right), y_{2}\right)$. Therefore we have that $\left(\psi^{-1}\left(h\left(x_{1}\right)\right), h\left(x_{2}\right)\right)=\left(y_{1}, y_{2}\right)$ and $\psi^{-1} \in A(X)$. Since $A(X)$ is abelian, it follows that $\left(h\left(\psi^{-1}\left(x_{1}\right)\right), h\left(x_{2}\right)\right)=\check{h}\left(\psi^{-1}\left(x_{1}\right), x_{2}\right)=\left(y_{1}, y_{2}\right)$, which proves that $\breve{h} \mathcal{R}(X, T)=\mathcal{R}(X, T)$.

For each $h \in A(X)$, we define the subsets $S_{h}(X)$ and $R_{h}(X)$ of $X \times X$ as follows:

$$
\begin{aligned}
S_{h}(X) & =\left\{\left(x, x^{\prime}\right) \in X \times X \mid\left(h(x), x^{\prime}\right) \in \mathcal{L}(X, T)\right\} \\
R_{h}(X) & =\left\{\left(x, x^{\prime}\right) \in X \times X \mid\left(h(x), x^{\prime}\right) \in \mathcal{P}(X, T)\right\} .
\end{aligned}
$$

Note that $S_{1_{X}}(X)=\mathcal{L}(X, T)$ and $R_{1_{X}}(X)=\mathcal{P}(X, T)$.

If $\mathcal{V}$ and $\mathcal{H}$ are relations in $X$, then $\mathcal{V} \circ \mathcal{H}$ is the relation in $X$ defined by as follows : $(z, y) \in \mathcal{V}$.

$(x, y) \in \mathcal{V} \circ \mathcal{H}$ if and only if for some element $z,(x, z) \in \mathcal{H}$ and

Lemma 3.15. Let $(X, T)$ be a transformation group and let $h \in A(X)$. Then $S_{h}(X) \neq \varnothing$ and $R_{h}(X) \neq \varnothing$.

Proof. Let $h, k \in A(X)$ and let $x^{\prime}=h(x)$. Then $\left(h(x), x^{\prime}\right) \in \triangle_{X} \subset$ $\mathcal{L}(X, T) \subset \mathcal{P}(X, T)$ by Lemma 3.1. Therefore $\left(x, x^{\prime}\right) \in S_{h}(X)$ and $\left(x, x^{\prime}\right) \in R_{h}(X)$. This proves that $S_{h}(X) \neq \varnothing$ and $R_{h}(X) \neq \varnothing$. 
Theorem 3.16. Suppose that $(X, T)$ is a transformation group and that $E(X)$ contains just one minimal right ideal. Then $R_{h}(X) \circ R_{k}(X)=$ $R_{h \circ k}(X)$ for all $h, k \in A(X)$.

Proof. Let $h, k \in A(X)$ and $(x, y) \in R_{h}(X) \circ R_{k}(X)$. Then there exists $z \in X$ such that $(x, z) \in R_{k}(X)$ and $(z, y) \in R_{h}(X)$. Hence $(k(x), z) \in \mathcal{P}(X, T)$ and $(h(z), y) \in \mathcal{P}(X, T)$. Therefore by Theorem 3.13(1) $(h(k(x)), h(z)) \in \mathcal{P}(X, T)$. Since $E(X)$ contains just one minimal right ideal, it follows from Remark 2.1 that $\mathcal{P}(X, T)$ is transitive and therefore $(h(k(x)), y) \in \mathcal{P}(X, T)$. Since $h \circ k \in A(X)$, we have that $(x, y) \in R_{h \circ k}(X)$.

Let $(x, y) \in R_{h \circ k}(X)$. By Theorem 3.13(1), $(h(k(x)), y) \in \mathcal{P}(X, T)$ shows that $\left(k(x), h^{-1}(y)\right) \in \mathcal{P}(X, T)$. Now let $h^{-1}(y)=z$. Then $(k(x), z)) \in \mathcal{P}(X, T)$ and $h(z)=y$. Since $(y, y) \in \mathcal{P}(X, T)$, it follows that $(h(z), y) \in \mathcal{P}(X, T)$. Hence $(x, z) \in R_{k}(X)$ and $(z, y) \in R_{h}(X)$. Thus $(x, y) \in R_{h}(X) \circ R_{k}(X)$.

The next corollary states that if $E(X)$ contains just one minimal right ideal, then $\left(\left\{R_{h}(X) \mid h \in A(X)\right\}, \circ\right)$ forms a group.

Corollary 3.17. Suppose that $(X, T)$ is a transformation group and that $E(X)$ contains just one minimal right ideal. For arbitrary $h, k, r \in A(X)$, the following properties hold:

(1) $\left(R_{h}(X) \circ R_{k}(X)\right) \circ R_{r}(X)=R_{h}(X) \circ\left(R_{k}(X) \circ R_{r}(X)\right)$.

(2) There exists $1_{X} \in A(X)$ such that $\mathcal{P}(X, T) \circ R_{h}(X)=R_{h}(X) \circ \mathcal{P}(X, T)=R_{h}(X)$.

(3) For each $h \in A(X)$ there exists $h^{-1} \in A(X)$ such that $R_{h}(X) \circ R_{h^{-1}}(X)=R_{h^{-1}}(X) \circ R_{h}(X)=\mathcal{P}(X, T)$.

Proof. This follows from Lemma 3.15, Theorem 3.16, and the fact that $A(X)$ is a group.

Corollary 3.18. Let $(X, T)$ be a transformation group. Then the following statements are true :

(1) $S_{h}(X) \circ S_{k}(X)=S_{h \circ k}(X)$ for all $h, k \in A(X)$.

(2) $\left(S_{h}(X) \circ S_{k}(X)\right) \circ S_{r}(X)=S_{h}(X) \circ\left(S_{k}(X) \circ S_{r}(X)\right)$ for all $h, k, r \in$ $A(X)$.

(3) $\mathcal{L}(X, T) \circ S_{h}(X)=S_{h}(X) \circ \mathcal{L}(X, T)=S_{h}(X)$ for all $h \in A(X)$.

(4) $\left(S_{h}(X)\right)^{-1}=S_{h^{-1}}(X)$ for all $h \in A(X)$. 
Proof. The proof of (1) is analogous to that of Theorem 3.16. Note that $S_{h}(X) \neq \varnothing$ and $\breve{h} \mathcal{L}(X, T) \subset \mathcal{L}(X, T)$ for all $h \in A(X)$, and $\mathcal{L}(X, T)$ is an invariant equivalence relation on $X$.

REMARK 3.19. (1)The collection $\left(\left\{S_{h}(X) \mid h \in A(X)\right\}\right.$, o) is a group by Corollary 3.18.

(2) Suppose $(X, T)$ is distal. The collection $\left(\left\{R_{h}(X) \mid h \in A(X)\right\}, \circ\right)$ forms a group because $(X, T)$ is distal iff $E(X)$ is a minimal right ideal (see [4, Proposition 5.3]).

\section{References}

[1] J. Auslander, Regular minimal sets 1, Trans. Amer. Math. Soc. 123 (1966), 469 479.

[2] I.U. Bronstein, Extension of minimal transformation groups, Sijthoff and Nordhoff Inter. Publ., Netherlands, 1979.

[3] J.P. Clay, Proximity relations in transformation groups, Trans. Amer. Math. Soc. 108 (1963), 88-96.

[4] R. Ellis, Lectures on topological dynamics, Benjamin, New York, 1969.

[5] _ A semigroup associated with a transformation group, Trans. Amer. Math. Soc. 94 (1960), 272-281.

[6] R. Ellis and W.H. Gottschalk, Homomorphisms of transformation groups, Trans. Amer. Math. Soc. 94 (1960), 258-271.

[7] S. Glasner, Compressibility properties in topological dynamics, Amer. J. Math. 97 (1975), 148-171.

[8] P.S. Shoenfeld, Regular homomorphisms of minimal sets, Doctoral Dissertation, Univ. of Maryland (1974).

[9] J.O. Yu, Regular relations in transformation group, J. Korean Math. Soc. 21 (1984), 41-48.

[10] , The regionally regular relations, J. Chungcheong Math. Soc. 19 (2006), $365-373$

Department of Mathematics

Kwangwoon University

Seoul 139-701, Korea

E-mail: songhs@kw.ac.kr 